\title{
Position-Dependent Effects of Fluorinated Amino Acids on the Hydrophobic Core Formation of a Heterodimeric Coiled Coil
}

\author{
Mario Salwiczek, ${ }^{[a]}$ Sergey Samsonov, ${ }^{[b]}$ Toni Vagt, ${ }^{[a]}$ Elisabeth Nyakatura,${ }^{[a]}$ \\ Emanuel Fleige, ${ }^{[a]}$ Jorge Numata, ${ }^{[c]}$ Helmut Cölfen, ${ }^{[d]}$ M. Teresa Pisabarro, ${ }^{*[b]}$ and \\ Beate Koksch*[a]
}

Dedicated to Professor Klaus Burger on the occasion of his 70th birthday

\begin{abstract}
Systematic model investigations of the molecular interactions of fluorinated amino acids within native protein environments substantially improve our understanding of the unique properties of these building blocks. A rationally designed heterodimeric coiled coil peptide (VPE/VPK) and nine variants containing amino acids with variable fluorine content in either position a16 or d19 within the hydrophobic core were synthesized and used
\end{abstract}

to evaluate the impact of fluorinated amino acid substitutions within different hydrophobic protein microenvironments. The structural and thermodynamic stability of the dimers were examined by applying both experimental (CD spectroscopy, FRET, and analyti-

Keywords: amino acids • fluorine • helical structures $\cdot$ molecular dynamics cal ultracentrifugation) and theoretical (MD simulations and MM-PBSA free energy calculations) methods. The coiled coil environment imposes position-dependent conformations onto the fluorinated side chains and thus affects their packing and relative orientation towards their native interaction partners. We find evidence that such packing effects exert a significant influence on the contribution of fluorine-induced polarity to coiled coil folding.

\section{Introduction}

[a] M. Salwiczek, ${ }^{+}$T. Vagt, ${ }^{+}$E. Nyakatura, ${ }^{+}$E. Fleige, ${ }^{+}$ Prof. Dr. B. Koksch

Department of Biology, Chemistry, and Pharmacy Freie Universiät Berlin

Institute of Chemistry and Biochemistry-Organic Chemistry Takustrasse 3, 14195 Berlin (Germany)

Fax: (+49)30-838-55644

E-mail: koksch@chemie.fu-berlin.de

[b] S. Samsonov," Dr. M. T. Pisabarro ${ }^{\#}$

Structural Bioinformatics, BIOTEC TU Dresden

Tatzberg 47-51, 01307 Dresden (Germany)

Fax: (+49)351-463-40087

E-mail: mayte.pisabarro@biotec.tu-dresden.de

[c] J. Numata

Department of Biology, Chemistry, and Pharmacy

Freie Universiät Berlin

Institute of Chemistry and Biochemistry-Crystallography

Takustrasse 6, 14195 Berlin (Germany)

[d] Dr. H. Cölfen

Max Planck Institute of Colloids and Interfaces

Am Mühlenberg 1, 14476 Potsdam-Golm (Germany)

$\left.{ }^{+}\right]$Experimental part of this work.

[\#] Theoretical part of this work.
The widespread interest in peptides and proteins as highly potent pharmaceuticals ${ }^{[1]}$ as well as bio-inspired materials ${ }^{[2]}$ motivates attempts towards the de novo design of peptides and proteins with superior properties such as chemical and metabolic resistance as well as thermodynamic stability. ${ }^{[3]}$ Moreover, endowing these biomolecules with novel functions that are not carried out by natural proteins ${ }^{[4]}$ is perhaps one of the most interesting, albeit challenging prospects in protein engineering. ${ }^{[5]}$ To this end, continuous efforts are made to expand the repertoire of genetically encoded amino acids through manipulation of the translational machinery in vitro and in vivo. ${ }^{[6,7]}$ Also, pure synthetic and semisynthetic approaches, that is, the direct chemical modification of protein functional groups ${ }^{[8]}$ as well as solid-phase peptide synthesis, ${ }^{[9]}$ native chemical ligation, ${ }^{[10]}$ and expressed protein ligation ${ }^{[11]}$ enable the incorporation of non-natural amino acids into peptide and protein sequences. In this context, fluorinated amino acids have increasingly gained recognition as analytical probes and modulators for protein structure and stability. ${ }^{[12]}$ 
Organic molecules containing $\mathrm{C}-\mathrm{F}$ bonds display unique properties $^{[13]}$ that account for their ever-growing importance in medicinal chemistry. ${ }^{[14]}$ Most prominent amongst these is a pronounced enhancement in steric size upon fluorination of alkyl groups that is combined with the very low polarizability of the fluorine atom. This often, although not generally, ${ }^{[15]}$ leads to a manifold increase in hydrophobicity and thus improves membrane permeability. ${ }^{[16]}$ It has been anticipated that global replacement of hydrophobic amino acids in hydrophobic domains with fluorinated analogues would accordingly stabilize the structure of proteins. As summarized in a recent review ${ }^{[17]}$ this has been proven to be a successful concept for the design of hyperstable $\alpha$-helical coiled coils. Along with enhanced self-association behavior, some of these peptides display an increase in membrane-binding affinity ${ }^{[18,19]}$ that lead to the design of fluorinated peptides with enhanced antimicrobial activity. ${ }^{[20,21]}$ The attempt towards a global replacement of leucine residues by fluorinated analogues within globular proteins, however, resulted in reduced thermodynamic stability. ${ }^{[2,23]}$ In these cases, additional mutations were needed to compensate for the disadvantageous effects. ${ }^{[24]}$ It was also shown that fluorination of aromatic side chains within proteins does not generally enhance secondary structure formation. ${ }^{[25,26]}$ These findings suggest that properties other than hydrophobicity may also play an important role in directing the interactions of fluorine within native protein environments. Though a weak electron donor and thus poor hydrogen-bond acceptor, ${ }^{[27]}$ carbon-bound fluorine has been shown to participate in favorable multipolar interactions within native protein environments. ${ }^{[28]}$ It is also important to note that despite the fact that specific fluorine-fluorine interactions are able to promote ordered self-association, ${ }^{[17]}$ it has been proposed that they may also result in misfolding. ${ }^{[22,29]}$ In addition, our previous studies suggest that hydrophobic interactions in proteins may be severely disturbed by fluorine-induced polarity. ${ }^{[29]}$ In summary, it still seems rather difficult to predict the impact of fluorination on the structure and activity of peptides and proteins. To further investigate the impact of fluorine substitution in native protein environments, we designed a heterodimeric $\alpha$-helical coiled coil peptide containing one fluorinated amino acid at either of two positions within the hydrophobic core, which are different in terms of side chain packing. We find that the effect of fluorine-induced polarity highly depends on the microenvironment of the substitution.

\section{Results and Discussion}

Peptide design: The aim of this study was to evaluate how fluorinated amino acids interact with native residues in a natural protein environment. A previously reported de novo designed $\alpha$-helical coiled coil interaction motif was shown to sufficiently fulfil the requirements for an appropriate model system. ${ }^{[29]}$ Besides being of paramount biological importance ${ }^{[30,31]}$ the coiled coil's greatest advantage is that it pro- vides two very well defined recognition surfaces. ${ }^{[32]}$ Its primary structure is based on a repetitive pattern of seven amino acids, the heptad repeat (abcdefg) $)_{n}$. Along the helical surface, the hydrophobic positions a and d and the mostly polar positions $\mathbf{b}, \mathbf{c}$, and $\mathbf{f}$ point in opposite directions. The $\mathbf{a}$ and $\mathbf{d}$ residues of the interacting helices are packed in a zipper-like fashion to form the hydrophobic core while all the other heptad positions are solvent exposed. The perfect interactions within the hydrophobic core provide the basis for a stable fold and drive oligomerization. In consequence the peptides associate to form a slightly left-handed superhelix. In dimeric coiled coils positions $\mathbf{e}$ and $\mathbf{g}$ are preferably populated by charged residues that further contribute to stability and control the specificity of folding by forming interhelical salt bridges. Following this primary structure code coiled coils of different length and oligomerization specificity can be designed de novo. ${ }^{[32]}$ Because the packing of the hydrophobic side chains in a parallel coiled coil, a against $\mathbf{a}^{\prime}$ and $\mathbf{d}$ against $\mathbf{d}^{\prime}$, is not equivalent in terms of relative side chain orientation (see below), ${ }^{[33]}$ a parallel design as presented below can be used to study the impact of fluorination within two different hydrophobic microenvironments.

The model system VPE/VPK was designed to provide the environment for specific interactions between a fluorinated and a non-fluorinated peptide. The peptide model fulfils two important criteria: 1) specificity for one distinct orientation of the peptide strands within the dimer and 2) heterodimerization. Figure 1 illustrates the design of the model peptide. The amino acid composition of the hydrophobic core is inspired by the GCN4 transcription factor, which has already been extensively characterized at high resolution. ${ }^{[34]}$ Here, valine in all a and leucine in all $\mathbf{d}$ positions provide for a parallel orientation of the peptide strands in the coiled coil dimer.

Most important for the purpose of the study, heterodimerization is required to guarantee that the observed effects trace back to a single fluoroamino acid substitution per dimer. This condition is accomplished by introducing $\mathbf{e}-\mathbf{g}^{\prime}$ and $\mathbf{g}-\mathbf{e}^{\prime}$ pairs that engage in favorable electrostatic interactions in the heterodimer but would repel one another in both possible homodimers. The fully natural VPE peptide was then used as a template to screen the interactions with different fluorine-containing variants of the complementary interaction partner VPK.

As mentioned above, the packing characteristics of the a and d positions in parallel coiled coils are different (see Figure 7). Therefore, the VPK strand contains the fluorinated amino acids either at position a16 or d19, which allows evaluating the impact of fluorination within two different hydrophobic microenvironments. Peptides containing leucine at the respective substitution site served as the reference peptides.

FRET and analytical ultracentrifugation: The parent peptides VPE and VPK were used to verify the parallel heterodimerization of the model system. In order to determine the relative orientation of the helices we applied a FRET assay 

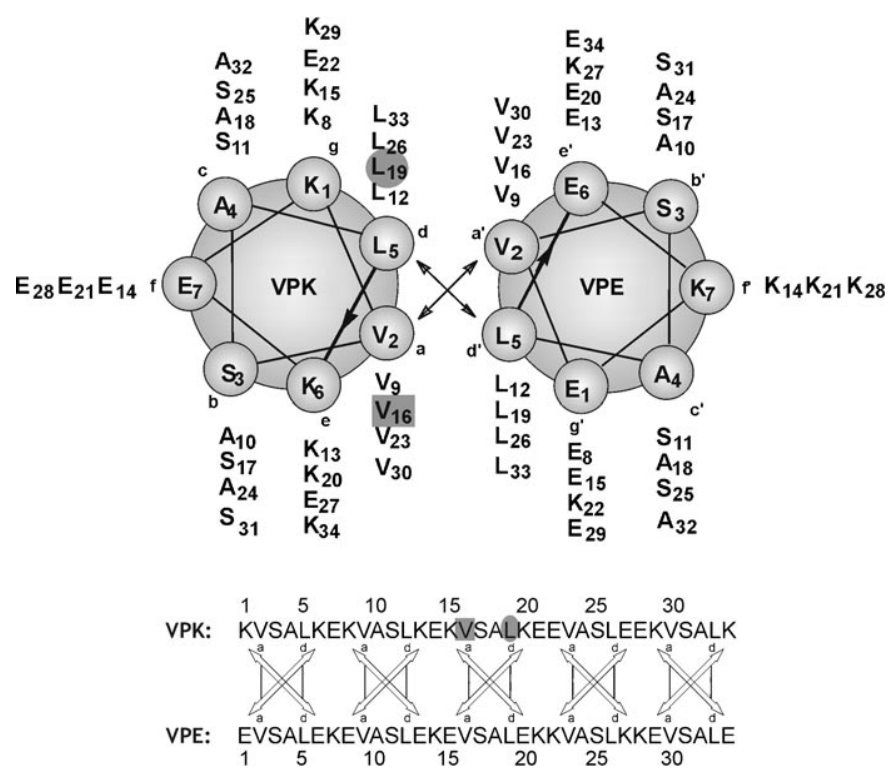

Figure 1. Amino acid sequence and helical wheel representation of the heterodimeric coiled coil model system. Two series of peptides were synthesized: fluorinated amino acid 1) at position a16 (grey box) and 2) at position d19 (grey circle) within VPK. Each peptide carries Abz at its Nterminus (not shown).

using $o$-aminobenzoic acid $(\mathrm{Abz})$ as the fluorescence donor and 3-nitrotyrosine $\left(\mathrm{YNO}_{2}\right)$ as the acceptor. ${ }^{[36]}$ Resonance energy transfer from $\mathrm{Abz}$ to $\mathrm{YNO}_{2}$ only occurs when the donor and the acceptor are in close proximity. For a parallel alignment, this condition is fulfiled when donor and acceptor are attached to the respective N-termini of VPK and VPE.

Figure $2 \mathrm{~A}$ shows the fluorescence spectra of N-terminally Abz-labeled VPK (VPK-NAbz, where Abz is attached to the N-terminus) at different concentrations of $\mathrm{N}$-terminally $\mathrm{YNO}_{2}$-labeled VPE $\left(\mathrm{VPE}-\mathrm{NYNO}_{2}\right)$. The spectra show a progressive decrease in fluorescence intensity as the concentration of VPE increases. A similar experiment in which the fluorescence donor $\mathrm{Abz}$ was present at the C-terminus of VPK (VPK-CAbz) shows much weaker quenching (Figure $2 \mathrm{~B}$ ) and confirms that VPE and VPK preferentially form parallel heterooligomers. Furthermore, control experiments in the presence of a denaturant $(\mathrm{GdnHCl})$ demonstrated that the quenching shown in Figure $2 \mathrm{~A}$ is the result of specific folding rather than self-quenching (see Supporting Information).

The oligomerization state of the VPE-VPK heterooligomers was determined by sedimentation velocity and equilibrium experiments. Sedimentation velocity experiments show artificial peak broadening due to insufficient removal of diffusion effects in the evaluation algorithm yielding a molar mass estimate of $7000 \mathrm{~g} \mathrm{~mol}^{-1}$ for the VPE-VPK heterodimer and a monomodal distribution confirming that only heterodimer is present in solution (Figure 3). This result was confirmed by the absolute molar mass determinations enabled by sedimentation equilibrium measurements, which yielded a $M_{\mathrm{w}}$ of $7600 \mathrm{~g} \mathrm{~mol}^{-1}$ from the extrapolation of five
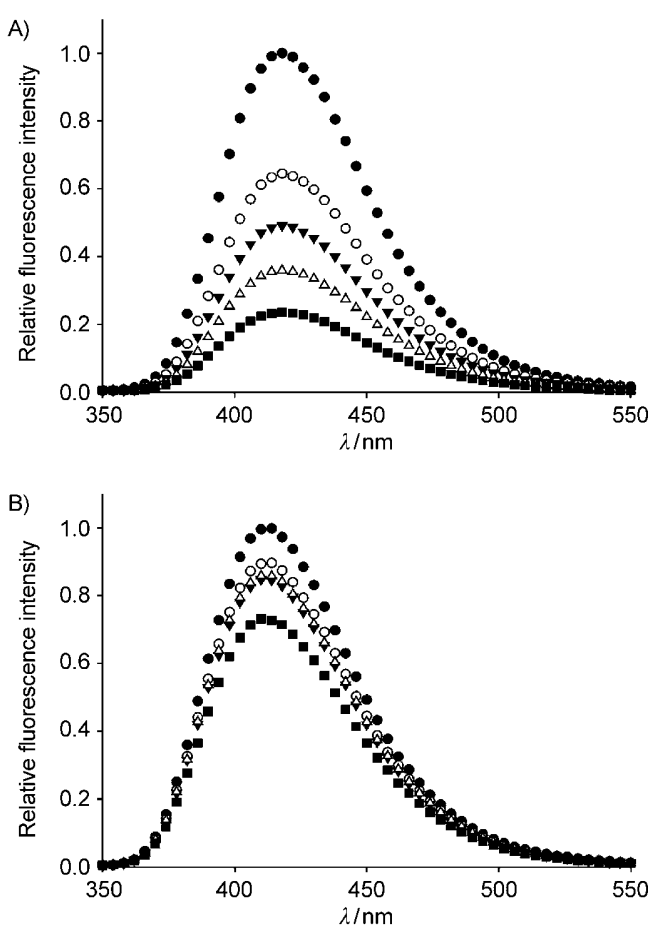

Figure 2. Normalized fluorescence spectra of A) $150 \mu \mathrm{g} \mathrm{mL}^{-1} \mathrm{VPK}-\mathrm{NAbz}$ at different concentrations of VPE-NYNO2 and B) $150 \mu \mathrm{g} \mathrm{mL}^{-1} \mathrm{VPK}-$ $C \mathrm{Abz}$ at different concentrations of $\mathrm{VPE}-\mathrm{NYNO}_{2}:(\bullet) 0 \mu \mathrm{gLL}^{-1}$, (०) $50 \mu \mathrm{g} \mathrm{mL}^{-1},(\boldsymbol{\nabla}) 100 \mu \mathrm{g} \mathrm{mL}^{-1},(\triangle) 150 \mu \mathrm{g} \mathrm{mL}^{-1}$, and $(\boldsymbol{\nabla}) 300 \mu \mathrm{g} \mathrm{mL}^{-1}\left(\lambda_{\mathrm{ex}}=\right.$ $320 \mathrm{~nm})$.

$M_{\text {w,app. }}$ to infinite dilution. This molar mass agrees very well with the expected molar mass for the VPE-VPK heterodimer of $7580.82 \mathrm{~g} \mathrm{~mol}^{-1}$ and confirms the specific heterodimerization of the model system. The formal extrapolation to infinite dilution is necessary to remove the effects of charge and excluded volume on the determined apparent molar mass, which is found too low with increasing concentration due to these non-ideal effects. Although at infinite dilution monomer is to be expected, this formal extrapolation was possible for the investigated concentration range of 100$500 \mu \mathrm{M}$, since Figure 3B shows the absence of association or dissociation of the heterodimer in this concentration range.

CD spectroscopy and MD simulations: All CD spectra of the equimolar mixtures of VPE and VPK-analogues display distinct minima at 208 and $222 \mathrm{~nm}$ at $20^{\circ} \mathrm{C}$ (Figure 4), indicating that all peptides form stable $\alpha$-helical structures. Also, the intensities for all heteromers are very similar, which suggests that the substitution of leucine by Abu and its fluorinated analogues (Scheme 1) at either position a16 or d19 only causes minor structural perturbations. We carried out MD simulations to verify these findings and further support our studies. The results of these experiments show that the structures of all heterodimeric coiled coils investigated here remain stable in solution. The root mean square deviation (RMSD) values for all atoms did not exceed $2.5 \AA$ (2.0 $\AA$ for backbone atoms). Moreover, the distances between $C_{\beta}$ atoms of the residues in $\mathbf{a}$ and $\mathbf{d}$ positions in each 

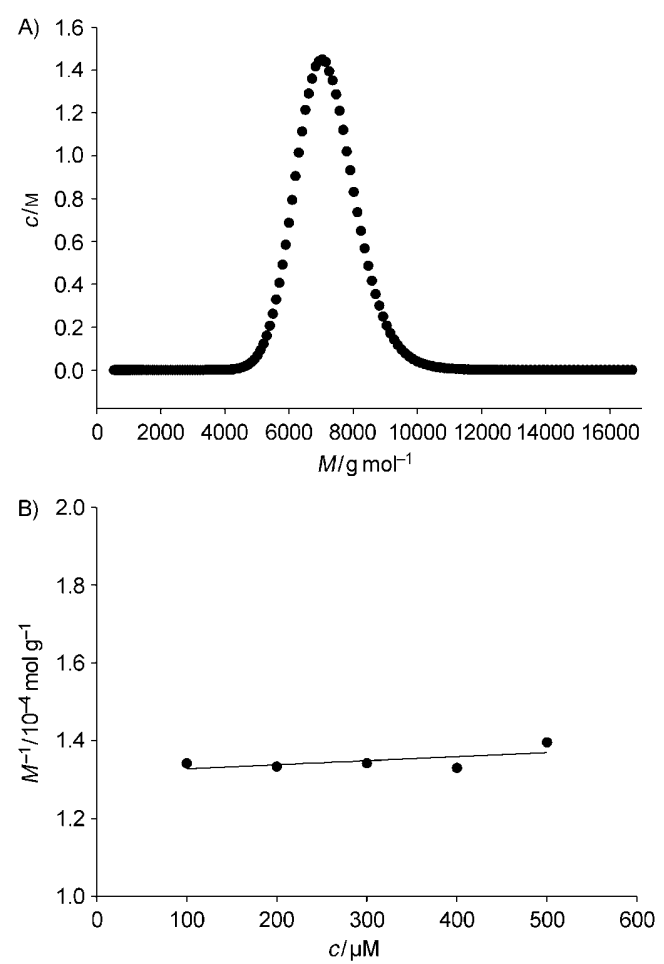

Figure 3. A) Diffusion corrected molar mass distribution $c(\mathrm{M})$ of the VPE-VPK heterodimer determined for a $50 \mu \mathrm{M}$ VPE-VPK sample. The peak is broadened due to insufficient removal of diffusion effects. B) Concentration dependence of the inverse apparent molar masses $M_{\text {w,app }}$. to yield $M_{\mathrm{w}}=7600 \mathrm{~g} \mathrm{~mol}^{-1}$ by formal extrapolation to infinite dilution (solid line).

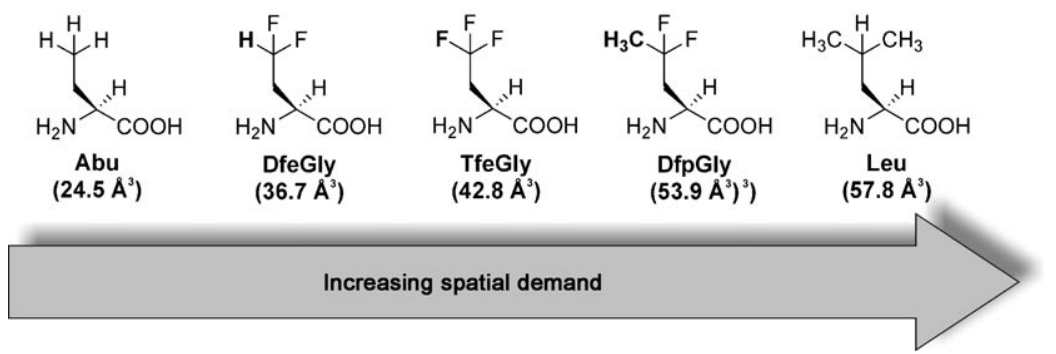

Scheme 1. Structures of $(S)$-aminobutyric acid (ethylglycine, Abu), $(S)$-4,4-difluoroethylglycine (DfeGly), $(S)$ 4,4,4-trifluoroethylglycine (TfeGly), $(S)$-4,4-difluoropropylglycine (DfpGly) and native leucine. The VdW volumes given in parentheses correspond to the alkyl groups that are attached to the $\beta$-carbon and were calculated according to Zhao et al. ${ }^{[35]}$

helix did not fluctuate substantially during simulation except for those at the N- and C-termini (Table S2, Supporting Information). The dihedral angles of all residues are comparable to values for an ideal $\alpha$-helix $\left(-60^{\circ}\right.$ and $-45^{\circ}$ for $\phi$ and $\psi$, respectively), again with the exception of the $\mathrm{N}$ - and $\mathrm{C}$ terminal residues. Although the coiled coil structure was preserved in all systems, small deviations from ideal $\alpha$-helical values were found for DfeGly16, DfeGly19 and Abu19. These results point to structural perturbations of the backbone that may, in part, account for the decreased thermodynamic stability of these dimers (see below).
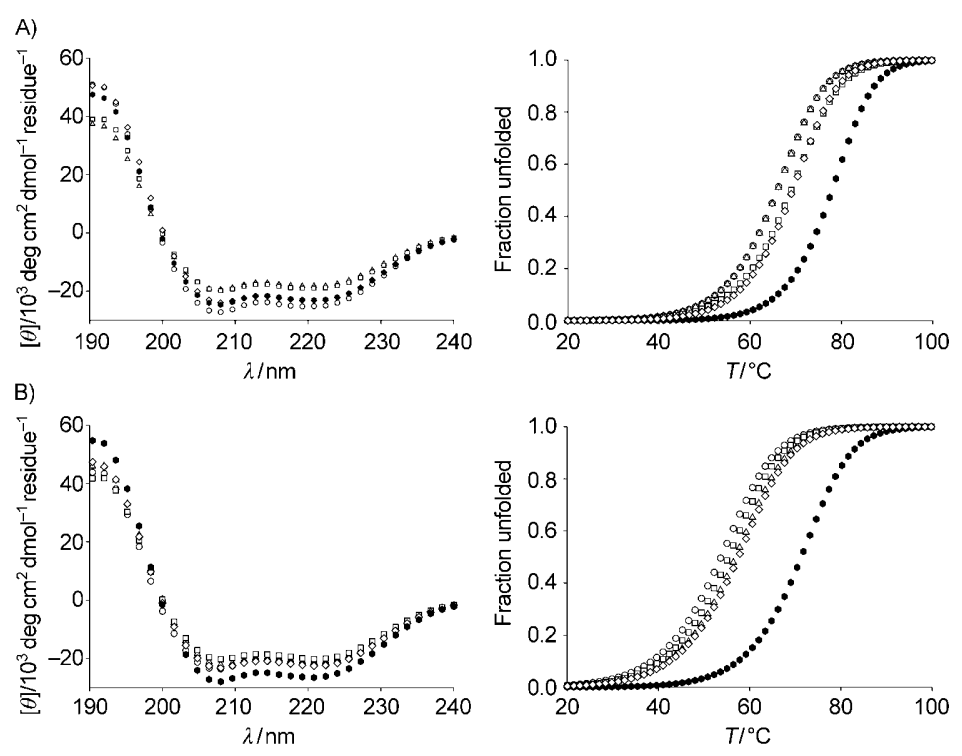

Figure 4. CD spectra at $20^{\circ} \mathrm{C}$ and fitted thermal unfolding profiles of the 1:1 VPE-VPK mixtures substituted at A) position $\mathbf{a 1 6}$ and B) position d19 of VPK: $(\bullet)$ Leu, (॰) Abu, $(\nabla)$ DfeGly, $(\square)$ TfeGly, and $(\diamond)$ DfpGly. Overall peptide concentrations were $20 \mu \mathrm{M}(10 \mu \mathrm{M}$ in each monomer at pH 7.4, 100 mм phosphate buffer).

Thermodynamic characterization: Temperature dependant circular dichroism spectroscopy was used to experimentally probe the thermodynamic stability of the dimers. All of them show cooperative thermal unfolding transitions upon heating from 20 to $100^{\circ} \mathrm{C}$ (Figure 4). The thermodynamic parameters of unfolding are summarized in Table 1. In both positions a16 and d19 the substitution of Leu by Abu and its fluorinated analogues considerably decreases the thermodynamic stability of the dimer. Comparison of the stabilities relative to leucine $\left(\Delta G^{\ominus}-\Delta G_{\text {Leu }}^{\ominus}\right)$, however, shows that in most cases substitution at position a16 seems to be less tolerated than substitution at position d19 (Figure 5). This loss in stability due to considerably removing hydrophobic surface area is partly attenuated by fluorination of the Abu side chain. Furthermore, while a pronounced increase in steric size of the fluorinated side chain by incorporation of DfpGly appears to further stabilize the folding motif at position a16 the same substitution at position d19 shows only marginal effects. Most strikingly, the findings for DfpGly contradict previous results for an antiparallel coiled coil model, where this residue as a replacement for leucine was found to disturb folding even stronger than alanine in an a position. ${ }^{[29]}$ 
Table 1. Thermodynamic parameters for the unfolding of the heterodimers substituted at position a16 and $\mathbf{d 1 9}$ of VPK.

\begin{tabular}{|c|c|c|c|c|}
\hline \multirow[b]{2}{*}{ Amino acid } & \multicolumn{2}{|c|}{ Position a16 } & \multicolumn{2}{|c|}{ Position d19 } \\
\hline & $\begin{array}{l}T_{\mathrm{m}} \\
{\left[{ }^{\circ} \mathrm{C}\right]^{[\mathrm{a}]}}\end{array}$ & $\begin{array}{l}\Delta G^{\odot} \\
{\left[\mathrm{kcal} \mathrm{mol}^{-1}\right]^{[\mathrm{b}]}}\end{array}$ & $\begin{array}{l}T_{\mathrm{m}} \\
{\left[{ }^{\circ} \mathrm{C}\right]^{[\mathrm{a}]}}\end{array}$ & $\begin{array}{l}\Delta G^{\ominus} \\
{\left[\mathrm{kcal} \mathrm{mol}^{-1}\right]^{[\mathrm{b}]}}\end{array}$ \\
\hline Leu & 77.9 & 13.8 & 71.3 & 11.7 \\
\hline $\mathrm{Abu}$ & 65.9 & 11.5 & 53.7 & 9.6 \\
\hline DfeGly & 66.9 & 11.5 & 56.9 & 10.0 \\
\hline TfeGly & 69.0 & 11.5 & 55.3 & 9.9 \\
\hline DfpGly & 69.3 & 12.3 & 57.5 & 10.0 \\
\hline
\end{tabular}

[a] $T_{\mathrm{m}}$ is defined as the temperature at which the fraction unfolded is 0.5 . Errors are typically not higher than $0.1^{\circ} \mathrm{C}$. [b] $\Delta G^{\circ}$ values were calculated for the $1 \mathrm{~m}$ standard state at $25^{\circ} \mathrm{C}$ using Equation (8). The value for $\Delta C_{\mathrm{p}}$ was determined from a Van't Hoff plot (see Supporting Information) to be $0.94 \pm 0.1 \mathrm{kcal} \mathrm{mol}^{-1} \mathrm{~K}^{-1}$. Errors for $\Delta G^{\circ}$ are typically not higher than $0.2 \mathrm{kcal} \mathrm{mol}^{-1}$ for the $\mathbf{a 1 6}$ and $0.1 \mathrm{kcal} \mathrm{mol}^{-1}$ for the $\mathbf{1 1 9}$ substituted peptides.

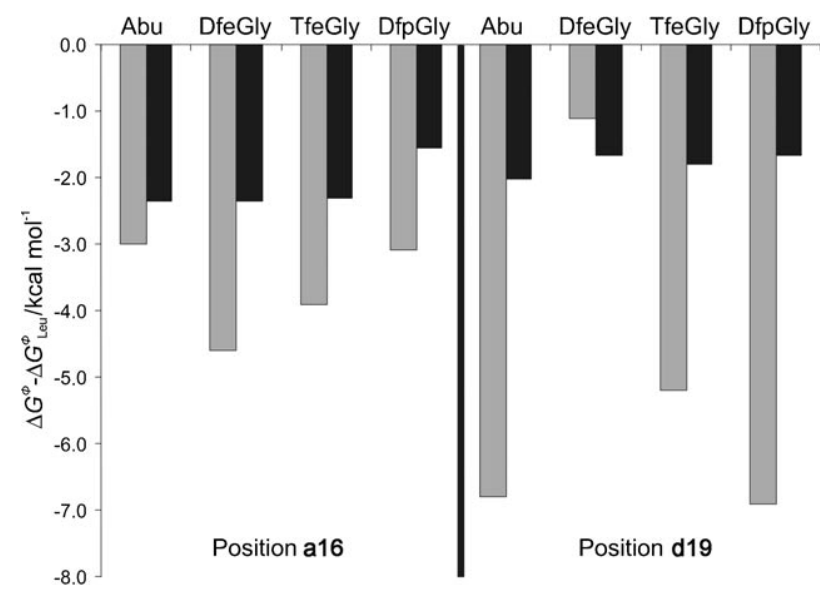

Figure 5. Relative stabilities of the a16- and d19-substituted dimers compared to the respective leucine variants as determined by thermal unfolding (black bars) and MM-PBSA analysis (grey bars).

The experimentally observed stability trends and those determined by the MM-PBSA energetic analysis are in agreement (Figure 5). The adjusted correlations between experimental and calculated enthalpies and free energies are 0.35 and 0.58 , respectively (with significance at the level of $p$ value 0.05 , Figure S5). Despite relatively low correlation coefficient values, our computational results also qualitatively distinguish substitutions at positions a16 and d19 (Figure 5 and Table S4). The theory supports the experimental finding that an increasing spatial demand of the fluorinated side chain at position a16 increases the stability of the dimer, while this trend is not reflected for identical substitutions at position d19. However, direct quantitative comparison of experimental and MM-PBSA data is not possible because of three factors: 1) there is an overestimation of the entropic component of the free energy because a single trajectory was used for the entropy calculation of the bound and unbound complex components, ${ }^{[37]}$ 2) the size of the model system was considerably reduced for the energy calculations, which results in a generally stronger impact on stability upon substitutions, and 3) entropy component are still the least accurate of MM-PBSA energy calculations. ${ }^{[38]}$

The general decrease in stability that was observed for the fluorinated peptides may be attributed to several factors. Recent investigations reveal that fluorine-containing amino acids exhibit weaker helix forming propensities than their native counterparts. ${ }^{[39]}$ Reliable thermodynamic scales for helix propensity are essentially measured using isolated helices. $^{[40]}$ Coiled coil stability, however, is substantially determined by interhelical interactions of the $\mathbf{a}$ and $\mathbf{d}$ positions within the hydrophobic core. ${ }^{[41]}$ For example, Abu in a monomeric helix favors helix formation by approximately $0.08 \mathrm{kcal} \mathrm{mol}^{-1[38]}$ compared to Leu but its substitution for Leu within the hydrophobic core of our coiled coil destabilizes the folding motif by more than $2 \mathrm{kcalmol}^{-1}$. Our MD simulations reveal mostly non-significant effects of fluorination on the conformational preferences of the amino acids within this coiled coil environment. We certainly do not rule out that introduction of fluorine affects helix propensity, but we would assign it less importance in the case of strongly interacting coiled coil residues. The stability of coiled coils generally correlates with hydrophobicity and with the spatial demand of hydrophobic side chains in positions $\mathbf{a}^{[42]}$ and d. ${ }^{[43]}$ In addition, the packing characteristics of side chains in both positions are significantly different (Figure 7). ${ }^{[33]}$ This difference may explain the general differences between relative stabilities of positions $\mathbf{a}$ and $\mathbf{d}$ as shown in Figure 5. The most striking dissimilarity between the positions is the relative orientation of the $\mathrm{C}_{\alpha}-\mathrm{C}_{\beta}$ vectors of interacting residues within the dimer. For a positions they point away from each other, whereas they point towards each other for $\mathbf{d}$ positions. Interestingly, this happens in all simulated coiled coil systems, suggesting a key role in the packing differences of $\mathbf{d}$ and $\mathbf{a}$ positions. For $\mathbf{a}$ and $\mathbf{d}$ positions the dihedral angles defined by both side chains (i.e., $\mathrm{C}_{\alpha}-\mathrm{C}_{\beta}-\mathrm{C}_{\beta}{ }^{\prime}-\mathrm{C}_{\alpha}{ }^{\prime}$ ) were found to be significantly different during the MD simulations $(-96 \pm$ 7 and $91 \pm 14^{\circ}$, respectively). Also there is an observable difference in $\mathrm{C}_{\beta}-\mathrm{C}_{\beta}^{\prime}$ distances, which is roughly $1 \AA$ shorter for d than for a positions (see Supporting Information). Figure 6 exemplarily illustrates the different packing for TfeGly at both substitution positions according to the MD simulations.

The fluorinated amino acids used in this study share a common structural feature, that is, fluorine substitution at the $\gamma$-carbon of the side chain, which results in a significant polarization of the $\beta$-methylene groups. According to the different packing characteristics at a and d positions described above, these $\beta$-methylene groups and their corresponding dipoles are closer to their hydrophobic interaction partners at the $\mathbf{d}$ than at the a positions. We conclude that fluorine-induced polarity may accordingly have varying degrees of importance for the stability of coiled coil interactions at these positions. Apparently, the impact is stronger at position $\mathbf{d 1 9}$ because, unlike for position a16, the increase in volume of the fluorinated side chains by methylation (DfpGly) is not able to gain further stability (see Figure 5 for experimental and Supporting Information for calcula- 


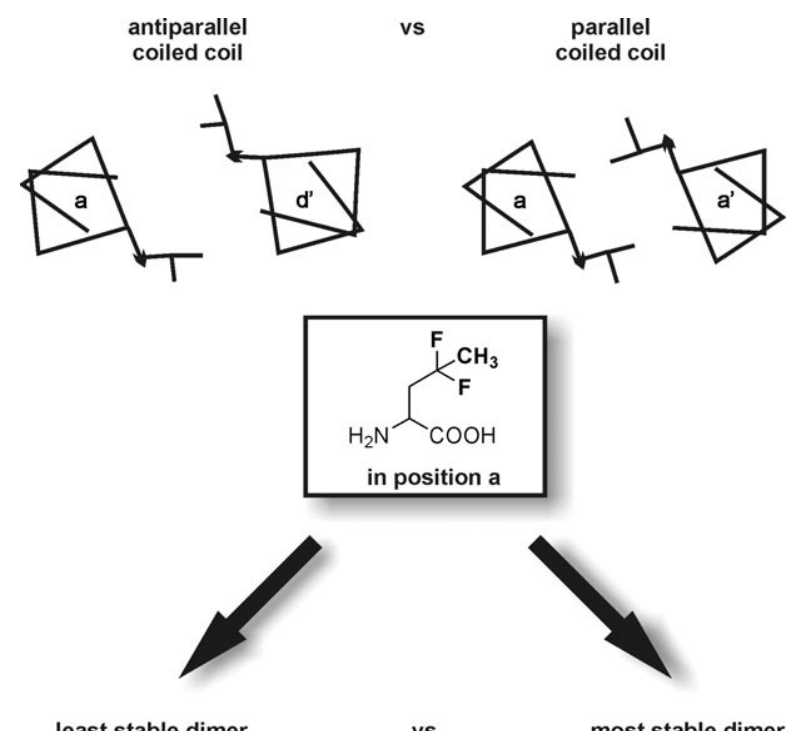

least stable dimer

vs

most stable dimer

Figure 6. Differences in packing of position $\mathbf{a}$ in antiparallel and parallel coiled coil dimers (according to ref. [32]) and consequences on the stability of DfpGly substitutions. Our findings for the parallel and the previously reported antiparallel system suggest that the orientation and flexibility of fluorinated side chains within a certain protein environment are additional factors that strongly determine the impact of fluorine-induced polarity. These conclusions are also supported by very recent MD studies by Pendley et al., which also reveal an important role of electrostatics in the stability of parallel coiled coil systems containing fluorinated amino acid residues $\left(5,5,5,5^{\prime} 5^{\prime} 5^{\prime}\right.$-hexafluoroleucine $)$ in the hydrophobic core. ${ }^{[44]}$
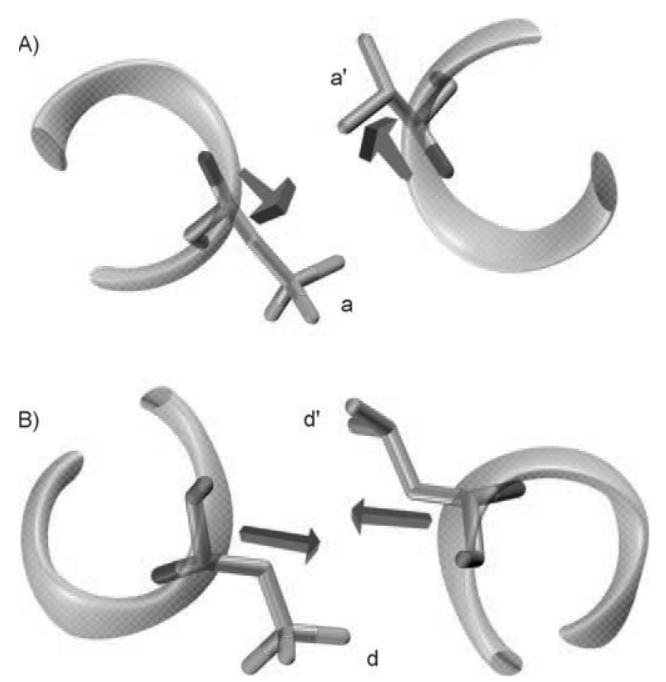

Figure 7. Packing of TfeGly against its direct interaction partner. A) position $\mathbf{a 1 6}$ and $\mathrm{B}$ ) position d19. The $\mathrm{C}_{\beta}$ atoms of the interacting side-chains are closer in the $\mathbf{d}$ position (B) than in the a position (A). The displayed $\mathrm{C}_{\alpha}-\mathrm{C}_{\beta}$ vectors highlight the significantly different packing characteristics of the side chains in a- and d- positions.

tions results). This is because the highly polarized $\beta$-methylene group in position $\mathbf{d 1 9}$ points towards the interaction partner in the opposite strand, while it points away from it at position a16. The interpretation that the impact of fluorine-induced polarity in amino acid side chains may depend on the packing and orientation of coiled coil helices gains further support from the finding that DfpGly in an antiparallel coiled coil ${ }^{[29]}$ destabilizes the folding motif much stronger than observed here (Figure 6). This is because the side chains in antiparallel coiled coils are generally more tightly buried within the core ${ }^{[33]}$ and we concluded that the highly polarized $\gamma$-methyl group of DfpGly strongly disturbs hydrophobic interactions. The differences in packing of position a in antiparallel and parallel coiled coil dimers are outlined in Figure 6 .

\section{Conclusion}

We have shown that the effect of fluorine at different positions within a heptad repeat on the stability of an $\alpha$-helical coiled coil can be rather ambiguous. Its effects highly depend on the microenvironment of a certain substitution that, in our case, is defined by both the substitution position and by helix orientation. Although the coiled coil model is a rather specific folding motif, our results imply that the packing and orientation of fluorinated side chains are very important in determining their interactions with native protein environments. The concept or notion that the introduction of fluorine into proteins necessarily leads to stabilization is clearly disputable according to our results. Changes in fluorine content and position of fluorination can considerably change the polarity and steric properties of an amino acid side chain and, thus, can influence the properties that a fluorinated amino acid develops within a native protein environment. This study shows that not only the fluorine itself, but also the characteristics of the environment determine the consequences of fluorine-induced polarity and steric demand of fluorinated side chains. Such systematic investigations will pave the way towards its directed application in protein engineering, for fine tuning of protein stability, their interactions with peptidic ligands as well as for therapeutical applications.

\section{Experimental Section}

Materials: Fmoc-Glu( $\mathrm{O} t \mathrm{Bu})-$ and Fmoc-Lys(Boc)-NovaSyn-TGA resins $\left(0,16 \mathrm{mmol} \mathrm{g}^{-1}\right.$ and $0,21 \mathrm{mmol} \mathrm{g}^{-1}$, respectively) were purchased from Novabiochem. Fmoc-L-amino acids, 2-(1H-benzotriazol-1-yl)-1,1,3,3-tetramethyluronium tetrafluoroborate (TBTU), and 1-hydroxybenzotriazole (HOBt) were purchased from Fa. Gerhardt (Wolfhagen, Germany). 1Hydroxy-7-azabenzotriazole (HOAt) was purchased from Iris Biotech, and Fmoc-protected $(S)$-2-aminobutyric acid (Abu) from Bachem. $(S)$-2Amino-4,4,4-trifluorobutyric acid (TfeGly), ${ }^{[45]}(S)$-2-amino-4,4-difluorobutyric acid (DfeGly), ${ }^{[46]}$ and $(S)$-2-amino-4,4-difluoropentanoic acid (DfpGly) ${ }^{[47]}$ were prepared according to literature procedures. Dimethylformamide (p.a., Acros), $N, N$-diisopropylethylamine (DIEA $98+\%$, Acros), $N, N$-diisoporopylcarbodiimide (DIC 99\%, Acros), trifluoroacetic acid (TFA 99\%, Acros), sodium perchlorate (p.a., Acros), triisopropylsilane (TIS 99\%, Acros), piperidine (99\% extra pure, Acros), acetonitrile (HPLC gradient grade, Acros), 1,8-diazabicyclo[5.4.0]undec-7-ene (for synthesis, Merck), disodium hydrogenphosphate dihydrate (p.a, Merck), and sodium dihydrogenphosphate dihydrate (ultra $>99 \%$, Fluka) were used without further purification. Acetic anhydride (99\%, Acros) was 
distilled prior to use. Deionized water for buffer solutions and HPLC was prepared using the MilliQ-AdvantageA10-System (Millipore). Water (solvent A) and acetonitrile (solvent B) for RP-HPLC were supplemented with $0.1 \%$ TFA (Uvasol, Merck)

Peptide synthesis, purification, and characterization: Peptides were synthesized using a SyroXP-I peptide synthesizer (MultiSynTech GmbH, Witten, Germany) on a $0.05 \mathrm{~mm}$ scale according to standard Fmoc/tBu chemistry ${ }^{[48]}$ For standard couplings a four fold excess of amino acids and coupling reagents (TCTU/HOBt) as well as an eight fold excess of DIEA relative to resin loading was used. All couplings were performed as double couplings $(30 \mathrm{~min})$. The coupling mixture contained $0.23 \mathrm{M}$ $\mathrm{NaClO}_{4}$ to prevent on-resin aggregation (see Supporting Information). Fluorinated amino acids as well as the first subsequent amino acid were activated by means of DIC/HOAt 1:1 protocols (seven minutes preactivation) without the addition of base to prevent racemization. ${ }^{[49]}$ The molar excess of amino acid and coupling reagents was reduced for fluorine-containing residues to 1.5 -fold for the first and 0.8 -fold for the second coupling. These couplings were performed manually until completion indicated by a negative Kaiser test. ${ }^{[50]}$ Prior to deprotection possibly non-acylated N-termini were capped by adding a mixture of acetic anhydride and DIEA $(10 \%$ each $)$ in DMF $(3 \times 10 \mathrm{~min})$. A mixture of DBU and piperidine $(2 \%$ each) in DMF was used for Fmoc deprotection $(4 \times 5 \mathrm{~min})$. Peptides were cleaved from the resin by treatment with $4 \mathrm{~mL}$ TFA/TIS/ $\mathrm{H}_{2} \mathrm{O}(95: 2.5: 2.5)$. The resins were washed twice with TFA $(1 \mathrm{~mL})$ and dichloromethane (dry, $1 \mathrm{~mL}$ ) and excess solvent was removed by evaporation. The peptides were precipitated with cold Et2O. Purification was carried out by RP-HPLC (Phenomenex Luna C8, $10 \mu \mathrm{m}, 250 \mathrm{~mm} \times 21.2 \mathrm{~mm}$ ) and the purity was confirmed by analytical HPLC (Phenomenex Luna $\mathrm{C} 8,5 \mu \mathrm{m}, 250 \mathrm{~mm} \times 4.6 \mathrm{~mm}$ ). All products were identified by high resolution ESI-MS (see Supporting Information). Peptide concentrations were determined using the absorbance of $o$-aminobenzoic acid $\left(\lambda_{\max }=320 \mathrm{~nm}\right.$ at $\mathrm{pH}$ 7.4) attached to the N-terminus of each peptide (see Supporting Information).

CD measurements: CD spectra were recorded on a Jasco J-715 spectropolarimeter at $20^{\circ} \mathrm{C}$ (Jasco PTC-348WI peltier thermostat). Overall peptide concentrations were $20 \mu \mathrm{M}(10 \mu \mathrm{M}$ VPE and $10 \mu \mathrm{M}$ VPK $)$ at $\mathrm{pH} 7.4$ (100 mm phosphate buffer). CD-spectra were obtained in the far-UV range (190-240 nm) using $0.1 \mathrm{~cm}$ Quartz Suprasil cuvettes (Hellma) equipped with a stopper. The nitrogen flow rate was set to $3 \mathrm{Lmin}^{-1}$. Ellipticity was normalized to concentration $\left(c\left[\mathrm{molL}^{-1}\right]\right)$, number of residues $(n=35$, including the N-terminal label) and path length $(l=1 \mathrm{~cm})$ using Equation (1):

$[\theta]=\theta_{\mathrm{obs}} /(10000 \cdot l \cdot c \cdot n)$

where $\theta_{\text {obs }}$ is the measured ellipticity in millidegrees and $[\theta]$ the normalized ellipticity in $10^{3} \mathrm{deg} \mathrm{cm}^{2} \mathrm{dmol}^{-1}$ residue ${ }^{-1}$. Melting curves were recorded using the signal at $222 \mathrm{~nm}$ applying a heating rate of $3 \mathrm{Kmin}^{-1}$ from 20 to $100^{\circ} \mathrm{C}$. Each sample was prepared three times and both the baseline corrected spectra and the melting curves were averaged.

FRET measurements: We carried out the FRET assay according to previously published procedures ${ }^{[51]}$ using $o$-aminobenzoic acid (Abz: $\lambda_{\mathrm{ex}}=$ $320 \mathrm{~nm}, \lambda_{\mathrm{em}}=420 \mathrm{~nm}$, Bachem) as the fluorescence label and 3-nitrotyrosine $\left(\mathrm{YNO}_{2}: \lambda_{\text {abs }}=420 \mathrm{~nm}\right.$, Bachem) as the quencher. ${ }^{[36]}$ Three peptides were synthesized: VPK carrying the Abz label at either the $\mathrm{N}$ - or the $\mathrm{C}$ terminus and VPE carrying $\mathrm{YNO}_{2}$ at the $\mathrm{N}$-terminus. Fluorescence spectra were recorded on a luminescence spectrometer LS 50B (Perkin Elmer) using a $1 \mathrm{~cm}$ Quartz Suprasil cuvette (Hellma) at $20^{\circ} \mathrm{C}$. Three scans from 350 to $550 \mathrm{~nm}$ were performed averaged and the spectra were normalized to the respective maximum fluorescence.

Calculation of thermodynamic parameters: Thermodynamic parameters were determined by non linear least square fitting of the normalized CDmelting curves to six parameters $\left(a, b,[\theta]_{\mathrm{M}}(0),[\theta]_{\mathrm{D}}(0), \Delta H_{\mathrm{m}}\right.$, and $\left.T_{\mathrm{m}}\right)$ assuming a two-state monomer-dimer equilibrium. The fits were performed in MS Excel as follows. Ellipticity can be calculated from the fraction unfolded $\left(f_{\mathrm{u}}\right)$ according to Equation (2):

$[\theta]=\left([\theta]_{\mathrm{M}}-[\theta]_{\mathrm{D}}\right) \cdot f_{\mathrm{u}}+[\theta]_{\mathrm{D}}$ where $[\theta]_{\mathrm{M}}$ represents the linear temperature dependence of the ellipticity of the fully unfolded monomers M [Eq. (3)], and VPK and VPE are mathematically regarded as equal. ${ }^{[52]}[\theta]_{\mathrm{D}}$ is the linear temperature dependance of the ellipticity of the fully folded dimer D [Eq. (4)]:

$[\theta]_{\mathrm{M}}=\mathrm{a} \cdot T+[\theta]_{\mathrm{M}}(0)$

$[\theta]_{\mathrm{D}}=\mathrm{a} \cdot T+[\theta]_{\mathrm{D}}(0)$

Here, $T$ is the temperature in ${ }^{\circ} \mathrm{C}$ and $[\theta]_{\mathrm{M}}(0)$ as well as $[\theta]_{\mathrm{D}}(0)$ represent the hypothetical ellipticity values for the unfolded and the folded peptides at $0{ }^{\circ} \mathrm{C}$. The fraction unfolded can be expressed in terms of equilibrium constant [Eq. (5)] after solving the equation for a bimolecular reaction $\mathrm{D} \rightleftarrows 2 \mathrm{M}$ :

$f_{\mathrm{u}}=\left\{\left(16 K[\mathrm{D}]_{0}+K^{2}\right)^{0.5}-\mathrm{K}\right\} / 8[\mathrm{D}]_{0}$

where $K$ is the equilibrium constant and $[\mathrm{D}]_{0}$ the concentration of the fully folded dimer. The temperature dependence of $K$ is expressed by Equation (6):

$K=\mathrm{e}^{-\Delta G / R T}$

The Gibbs-Helmholtz equation can be used to express the temperature dependence of $\Delta G$ in terms of $\Delta H_{\mathrm{m}}$ and $T_{\mathrm{m}}$ as given by Equation (7):

$\Delta G=\Delta H_{\mathrm{m}} \cdot\left(1-T / T_{\mathrm{m}}\right)+\Delta C_{\mathrm{p}} \cdot\left\{T-T_{\mathrm{m}}-T \cdot \ln \left(T / T_{\mathrm{m}}\right)\right\}$

where $\Delta H_{\mathrm{m}}$ is the enthalpy change at the melting temperature $T_{\mathrm{m}}$, that is defined as the temperature at which $f_{\mathrm{u}}=0.5 . \Delta C_{\mathrm{p}}$ is the change in heat capacity that was initially assumed to be zero for the purpose of fitting because due to the high interdependence of $\Delta H$ and $\Delta C_{\mathrm{p}}$ these parameters cannot be fitted simultaneously. Equations (2) through (7) were combined and the data fitted directly. $\Delta C_{\mathrm{p}}$ was calculated afterwards from the dependence of $\Delta H_{\mathrm{m}}$ from $T_{\mathrm{m}}$ and the standard free energy of unfolding $\Delta G^{\curvearrowright}$ (1 M standard state) was then calculated at $25^{\circ} \mathrm{C}$ according to Equation (8):

$\Delta G^{\curvearrowright}=\Delta H_{\mathrm{m}} \cdot\left(1-T / T_{\mathrm{m}}\right)+\Delta C_{\mathrm{p}} \cdot\left\{T-T_{\mathrm{m}}-T \cdot \ln \left(T / T_{\mathrm{m}}\right)\right\}-R T \cdot \ln 2[\mathrm{D}]_{0}$

Errors were determined by a statistical analysis of the fitted parameters. ${ }^{[53]}$ The error for the free energy of unfolding was calculated using Equation (8) applying the minimum and maximum values for $\Delta H_{\mathrm{m}}, \Delta C_{\mathrm{p}}$, and $T_{\mathrm{m}}$ according to their individual errors. To prove the validity of the fit, $\Delta H_{\mathrm{m}}$ and $T_{\mathrm{m}}$ were also determined manually using the Van't Hoff equation $^{[54]}$ (see Supporting Information).

Analytical ultracentrifugation: Analytical ultracentrifugation (AUC) was performed on a XL-I (Beckman-Coulter, Palo Alto, CA) ultracentrifuge at $25^{\circ} \mathrm{C}$ applying the UV/Vis absorption optics at $320 \mathrm{~nm}$ and using standard $12 \mathrm{~mm}$ double sector center pieces. Sedimentation velocity experiments were performed at $60000 \mathrm{rpm}$ and a sample concentration of $50 \mu \mathrm{M}$, sedimentation equilibrium experiments at $40000 \mathrm{rpm}$. The samples were dissolved in $100 \mathrm{~mm}$ phosphate buffer at pH 7.4 ( $\rho=$ $1.009942 \mathrm{~g} \mathrm{~mL}^{-1}, \eta=0.9243 \mathrm{cP}$ both at $25^{\circ} \mathrm{C}$ ). The partial specific volume of the samples was determined in a density oscillation tube (DMA 5000, Anton Paar, Graz) to be $0.730 \mathrm{mLg}^{-1}$ for VPK and $0.594 \mathrm{mLg}^{-1}$ for VPE. The partial specific volume of the VPE-VPK heterodimer was selected as the arithmetic average to be $0.662 \mathrm{mLg}^{-1}$. Apparent weight average molar masses were determined concentration dependent from sedimentation equilibrium experiments using the model independent MSTAR approach. ${ }^{[55]}$ Sedimentation velocity data were evaluated using the program SEDFIT by $\mathrm{P}$. Schuck ${ }^{[56]}$ yielding the diffusion corrected molar mass distribution $c(\mathrm{M})$.

MD simulations and MM-PBSA free energy calculations: The crystal structure of the Sir4p C-terminal coiled coil at $2.5 \AA$ resolution (1 PL5 PDB Id code) was used as template for modeling our parallel coiled coil systems. To obtain the parent peptide model system (VPE-VPK) and its 
nine fluorine-substituted variants the length of the helices of the Sir4p coiled coil was reduced to 34 aa, and the necessary side chain substitutions were carried out with the MOE program. ${ }^{[57]}$ The structures were solvated in a TIP3P water octahedral box, and periodic boundary conditions under constant temperature $(300 \mathrm{~K})$ and constant pressure $\left(10^{6} \mathrm{~Pa}\right.$ NTP) were applied. MD productive runs of simulations of $5 \mathrm{~ns}$ were performed with AMBER 8.0 $0^{[58]}$ using the ff03 force field. Non-standard amino acid residues were parameterized to be compatible with the Cornell force field using a standard procedure for non-natural amino acids $^{[59-62]}$ in the R.E.M. III program, which we used for RESP charge calculations. ${ }^{[63]}$ For each amino acid charges were derived for two conformations (helical and extended) with the ab initio Hartree-Fock method HF/6-31G* using GAMESS-US ${ }^{[64]}$ (the authors can provide derived charges information upon request). Energetic post-processing of the trajectories was done in a continuous solvent model as implemented in the AMBER 8.0 MM-PBSA module. The snapshots for the calculations were chosen as described by Lafont and co-workers. ${ }^{[65]}$ Entropies were calculated using normal mode analysis. Significant comparison of the free energies of interaction between two coiled coils is not possible because of the intrinsic flexibility of the helices termini. To avoid this additional source of noise in the MM-PBSA calculations only the central parts of the helices were analyzed (residues 10 to 25 , Table S2). Thus, taking into account the reduced size of our model system, only the comparison of relative values of energies with experimental data is reasonable. See Supporting Information for further details

\section{Acknowledgements}

We are grateful to the Deutsche Forschungsgemeinschaft (KO 1976/2-1 and Graduiertenkolleg \#788) and the Klaus Tschira Stiftung GmbH for financial support. We would also like to thank Antje Voelkl (MPI, Potsdam-Golm) for her very kind assistance with the ultracentrifugation experiments and Allison Berger for proofreading of the manuscript.

[1] A. K. Sato, M. Viswanathan, R. B. Kent, C. R. Wood, Curr. Opin. Biotechnol. 2006, 17, 638-642.

[2] K. Sanford, M. Kumar, Curr. Opin. Biotechnol. 2005, 16, 416-421.

[3] C. Baldauf, M. T. Pisabarro, J. Phys. Chem. B 2008, 112, 7581-7591.

[4] H. Zhao, Biotechnol. Bioeng. 2007, 98, 313-317.

[5] J. A. Brannigan, A. J. Wilkinson, Nat. Rev. Mol. Cell Biol. 2002, 3, 964-970.

[6] N. Budisa, Angew. Chem. 2004, 116, 6586-6624; Angew. Chem. Int. Ed. 2004, 43, 6426-6463.

[7] L. Wang, P. G. Schultz, Angew. Chem. 2005, 117, 34-68; Angew. Chem. Int. Ed. 2005, 44, 34-66.

[8] B. G. Davis, Curr. Opin. Biotechnol. 2003, 14, 379-386.

[9] W. C. Chan, P. D. White, Fmoc solid phase peptide synthesis: a practical approach, Oxford University Press, New York, 2000.

[10] J. P. Tam, J. Xu, K. D. Eom, Pept. Sci. 2001, 60, 194-205.

[11] R. David, M. P. Richter, A. G. Beck-Sickinger, Eur. J. Biochem. 2004, 271, 663-677.

[12] C. Jäckel, B. Koksch, Eur. J. Org. Chem. 2005, 4483-4503.

[13] D. O'Hagan, Chem. Soc. Rev. 2008, 37, 308-319.

[14] S. Purser, P. R. Moore, S. Swallow, V. Gouverneur, Chem. Soc. Rev. 2008, 37, 320-330.

[15] B. E. Smart, J. Fluorine Chem. 2001, 109, 3-11.

[16] G. Gerebtzoff, X. Li-Blatter, H. Fischer, A. Frentzel, A. Seelig, ChemBioChem 2004, 5, 676-684.

[17] M. Hakelberg, B. Koksch, Chemistry Today 2007, 25, 48-53.

[18] A. Niemz, D. A. Tirrell, J. Am. Chem. Soc. 2001, 123, 7407-7413.

[19] N. Naarmann, B. Bilgicer, H. Meng, K. Kumar, C. Steinem, Angew. Chem. 2006, 118, 2650-2653; Angew. Chem. Int. Ed. 2006, 45, 25882591.

[20] H. Meng, K. Kumar, J. Am. Chem. Soc. 2007, 129, 15615-15622.

[21] L. M. Gottler, H. Y. Lee, C. E. Shelburne, A. Ramamoorthy, E. N. Marsh, ChemBioChem 2008, 9, 370-373.
[22] T. Panchenko, W. W. Zhu, J. K. Montclare, Biotechnol. Bioeng. 2006 94, $921-930$.

[23] T. H. Yoo, A. J. Link, D. A. Tirrell, Proc. Natl. Acad. Sci. USA 2007, 104, 13887-13890.

[24] N. Voloshchuk, M. X. Lee, W. W. Zhu, I. C. Tanrikulu, J. K. Montclare, Bioorg. Med. Chem. Lett. 2007, 17, 5907-5911.

[25] M. G. Woll, E. B. Hadley, S. Mecozzi, S. H. Gellman, J. Am. Chem. Soc. 2006, 128, 15932-15933.

[26] C. Minks, R. Huber, L. Moroder, N. Budisa, Biochemistry 1999, 38, 10649-10659.

[27] J. D. Dunitz, R. Taylor, Chem. Eur. J. 1997, 3, 89-98.

[28] J. A. Olsen, D. W. Banner, P. Seiler, B. Wagner, T. Tschopp, U. Obst-Sander, M. Kansy, K. Müller, F. Diederich, ChemBioChem 2004, 5, 666-675.

[29] a) C. Jäckel, M. Salwiczek, B. Koksch, Angew. Chem. 2006, 118 , 4305-4309; Angew. Chem. Int. Ed. 2006, 45, 4198-4203; b) C. Jäckel, W. Seufert, B. Koskch, ChemBioChem 2004, 5, 717-720.

[30] J. M. Mason, K. M. Arndt, ChemBioChem 2004, 5, 170-176.

[31] A. Rose, I. Meier, Cell. Mol. Life Sci. 2004, 61, 1996-2009.

[32] D. N. Woolfson, Adv. Protein Chem. 2005, 70, 79-112.

[33] O. D. Monera, N. E. Zhou, C. M. Kay, R. S. Hodges, J. Biol. Chem. 1993, 268, 19218-19227.

[34] E. K. O’Shea, J. D. Klemm, P. S. Kim, T. Alber, Science 1991, 254, $539-544$.

[35] Y. H. Zhao, M. H. Abraham, A. M. Zissimos, J. Org. Chem. 2003, 68, 7368-7373.

[36] K. Pagel, K. Seeger, B. Seiwert, A. Villa, A. E. Mark, S. Berger, B. Koksch, Org. Biomol. Chem. 2005, 3, 1189-1194; For a general review of FRET in Bioanalysis, see I. L. Medintz, Angew. Chem. 2006, 118, 4676-4704; Angew. Chem. Int. Ed. 2006, 45, 4562-4588, and references therein.

[37] J. Wang, P. Morin, W. Wang, P. A. Kollman, J. Am. Chem. Soc. 2001, $123,5221-5230$.

[38] A. Weis, K. Katebzadeh, P. Soderhjelm, I. Nilsson, U. Ryde, J. Med. Chem. 2006, 49, 6596-6606.

[39] H. P. Chiu, Y. Suzuki, D. Gullickson, R. Ahmad, B. Kokona, R. Fairman, R. P. Cheng, J. Am. Chem. Soc. 2006, 128, 15556-15557.

[40] L. Regan, Proc. Natl. Acad. Sci. USA 1997, 94, 2796-2797.

[41] S. C. Kwok, C. D. Mant, R. S. Hodges in 25th European Peptide Symposium (Eds.: S. Bajusz, F. Hudecz), Akadémiai Kiadó, Budapest, 1998, pp. 34-35.

[42] K. Wagschal, B. Tripet, P. Lavigne, C. Mant, R. S. Hodges, Protein Sci. 1999, 8, 2312-2329.

[43] B. Tripet, K. Wagschal, P. Lavigne, C. T. Mant, R. S. Hodges, J. Mol. Biol. 2000, 300, 377-402.

[44] S. S. Pendley, Y. B. Yu, T. E. Cheatham III, Proteins Struct. Funct. Bioinf. 2008, 74, 612-629.

[45] T. Tsushima, K. Kawada, S. Ishihara, N. Uchida, O. Shiratori, J. Higaki, M. Hirata, Tetrahedron 1988, 44, 5375-5387.

[46] D. Winkler, K. Burger, Synthesis 1996, 1419-1421.

[47] S. N. Osipov, T. Lange, P. Tsouker, J. Spengler, L. Hennig, B. Koksch, S. Berger, S. M. El-Kousy, K. Burger, Synthesis 2004, 18211829.

[48] G. B. Fields, R. L. Noble, Int. J. Pept. Proetin Res. 1990, 35, 161-214.

[49] L. A. Carpino, A. El-Faham, Tetrahedron 1999, 55, 6813-6830.

[50] E. Kaiser, R. L. Colescott, C. D. Bossinger, P. I. Cook, Anal. Biochem. 1970, 34, 595-598.

[51] C. García-Echeverría, Bioorg. Med. Chem. Lett. 1997, 7, 1695-1698.

[52] D. Krylov, I. Mikhailenko, C. Vinson, EMBO J. 1994, 13, 28492861.

[53] E. J. Billo, Excel for Scientists and Engineers-Numerical Methods, Wiley, New York, 2007.

[54] L. A. Marky, K. J. Breslauer, Biopolymers 1987, 26, 1601-1620.

[55] H. Cölfen, S. E. Harding, Eur. Biophys. J. 1997, 25, 333-346.

[56] P. Schuck, Biophys. J. 2000, 78, 1606-1619.

[57] Molecular operating environment, Chemical Computing Group Inc., 2005.

[58] D. A. Case, T. A. Darden, T. E. Cheatham III, C. L. Simmerling, J. Wang, R. E. Duke, R. Luo, K. M. Merz, B. Wang, D. A. Pearlman, 
M. Crowley, S. Brozell, V. Tsui, H. Gohlke, J. Mongan, V. Hornak, G. Cui, P. Beroza, C. Schafmeister, J. W. Caldwell, W. S. Ross, P. A. Kollman, AMBER 8, 2004.

[59] P. Hobza, M. Kabelác, J. Sponer, P. Mejzilik, J. Vondrasek, J. Comput. Chem. 1997, 18, 1136-1150.

[60] S. A. Showalter, R. Bruschweiler, J. Am. Chem. Soc. 2007, 129, 4158-4159.

[61] D. L. Mobley, E. Dumont, J. D. Chodera, K. A. Dill, J. Phys. Chem. B 2007, 111, 2242-2254.

[62] A. Nicholls, D. L. Mobley, J. P. Guthrie, J. D. Chodera, C. I. Bayly, M. D. Cooper, V. S. Pande, J. Med. Chem. 2008, 51, 769-779.
[63] A. Pigache, P. Cieplak, F.-Y. Dupradeau in 227th ACS National Meeting, Anaheim, USA, 2004.

[64] M. W. Schmidt, K. K. Baldridge, J. A. Boatz, S. T. Elbert, M. S Gordon, J. H. Jensen, S. Koseki, N. Matsunaga, K. A. Nguyen, S. Su, T. L. Windus, M. Dupuis, J. A. Montgomery, J. Comput. Chem. 1993, 14, 1347-1363.

[65] V. Lafont, M. Schaefer, R. H. Stote, D. Altschuh, A. Dejaegere, Proteins Struct. Funct. Bioinf. 2007, 67, 418-434. 